\title{
Hydrothermal synthesis of nitrogen-doped carbon dots with real-time live-cell imaging and blood-brain barrier penetration capabilities
}

This article was published in the following Dove Press journal:

International Journal of Nanomedicine

28 November 2016

Number of times this article has been viewed

\section{Shousi Lu ${ }^{1,2}$ \\ Shanshan Guo' \\ Pingxiang $\mathrm{Xu}^{\prime}$ \\ Xiaorong $\mathrm{Li}^{\prime}$ \\ Yuming Zhao' \\ Wei $\mathrm{Gu}^{3}$ \\ Ming Xue}

'Department of Pharmacology, Beijing Laboratory for Biomedical

Detection Technology and Instrument,

School of Basic Medical Sciences,

Capital Medical University, ${ }^{2}$ China

Rehabilitation Research Center

${ }^{3}$ Department of Chemistry and

Biology, School of Chemical Biology

and Pharmaceutical Sciences, Capital

Medical University, Beijing, China
Correspondence: Wei Gu

School of Chemical Biology and

Pharmaceutical Sciences, Capital

Medical University, Number 10

You'anmenwaixitoutiao, Beijing 100069 ,

China

Tel +86 I0 839I I525

Email weigu@ccmu.edu.cn

Ming Xue

Department of Pharmacology, Beijing Laboratory for Biomedical Detection

Technology and Instrument, School of Basic Medical Sciences, Capital Medical University, Number 10 You'anmenwaixitoutiao, Beijing 100069, China

Tel/fax +86 I0 839I I520

Email xuem@ccum.edu.cn

\begin{abstract}
Nitrogen-doped carbon dots (N-CDs) were synthesized using a one-pot hydrothermal treatment with citric acid in the presence of polyethylenimine. Transmission electron microscopy analysis revealed that the N-CDs were monodispersed and quasi-spherical with an average size of $\sim 2.6 \mathrm{~nm}$. Under ultraviolet irradiation the N-CDs emitted a strong blue luminescence with a quantum yield as high as $51 \%$. Moreover, the N-CDs exhibited a negligible cytotoxicity and could be applied as efficient nanoprobes for real-time imaging of live cells. In addition, the ability of the N-CDs to cross the blood-brain barrier (BBB) in a concentration-dependent manner was demonstrated using an in vitro BBB model. Therefore, these PEI-passivated N-CDs with real-time live-cell imaging and BBB-penetration capabilities hold promise for traceable drug delivery to the brain.
\end{abstract}

Keywords: hydrothermal synthesis, nitrogen-doped carbon dots, bioimaging

\section{Introduction}

Carbon dots (CDs) are emerging as superior luminescent nanomaterials as they possess a high aqueous solubility, outstanding photoluminescence (PL) properties, favorable biocompatibility, and chemical inertness and are easily functionalized. ${ }^{1-4}$ As such, CDs have considerable advantages in cell labeling and bioimaging over semiconductor quantum dots (QDs) and conventional organic dyes. Nevertheless, the quantum yield (QY) of CDs is far lower than that of QDs, ${ }^{5,6}$ limiting their applications in cell labeling and bioimaging.

Doping of heteroatoms such as nitrogen atoms, in conjunction with appreciable surface passivation, has been proven to be an effective way to enhance the QY of CDs. ${ }^{5}$ So far, a variety of methods has been employed to synthesize nitrogen-doped carbon dots (N-CDs). In particular, the hydrothermal method is extremely attractive because of advantages such as relatively mild reaction conditions, inexpensive equipment, ${ }^{7}$ and the potential for functionalization. ${ }^{8}$ For example, N-CDs with QYs of $17.6 \%$ and $18.98 \%$ have been prepared by hydrothermal treatment of spider silk and papaya powder, respectively. ${ }^{9,10}$ However, such QYs are still less than those of QDs. Therefore, it remains highly desirable to produce N-CDs with an enhanced QY by hydrothermal methods.

This study aims to fabricate N-CDs with an enhanced QY via a hydrothermal method. This was accomplished by hydrothermal treatment of citric acid (CA) in the presence of polyethylenimine (PEI; Figure 1). In addition to acting as a nitrogen dopant, PEI simultaneously serves as a surface passivation agent to further improve 
the QY. The as-prepared PEI-passivated N-CDs could emit a strong blue luminescence, and the QY was up to $51 \%$, which is comparable to that of QDs. These N-CDs, with a high QY, are particularly useful for sensitive biosensing and bioimaging applications. ${ }^{11}$

CDs have also attracted increasing attention for their promising applications in drug delivery. ${ }^{12,13}$ For instance, Wang et al used CDs as a drug delivery system for doxorubicin (DOX), which produced a pH-controlled DOX release. ${ }^{14}$ Recently, an in vitro study demonstrated that CDs can potently inhibit insulin fibrillation, which is associated with neurological diseases. ${ }^{15}$ However, it remains a major medical challenge to deliver drugs to the brain, as a result of the presence of the blood-brain barrier (BBB), which exhibits tight junctions between endothelial cells, which inhibit the passage of therapeutic agents to pathological tissues in the brain. Fortunately, the tight junctions in the BBB possess a gap of 4-6 nm. Thus, it is possible that nanoparticles with a size $<4 \mathrm{~nm}$, such as CDs, could pass through the BBB via such gaps. ${ }^{16}$ Another important factor in nanoparticle BBB penetration is their electrostatic charge. It has been suggested that cationic molecules might occupy anionic areas at the BBB endothelium and thus produce an increased endothelial cell permeability. ${ }^{17}$ As a cationic macromolecule, $P E I$ renders CDs with a positive surface charge under physiological conditions, which could improve the biomembrane permeabilities. ${ }^{18}$ In this context, the BBB-penetration ability of PEI-passivated N-CDs was investigated using an in vitro biomimetic BBB model. ${ }^{19}$

\section{Experimental}

\section{Materials}

CA (Sinopharm Chemical Reagent Co. Ltd, Beijing, China), PEI (molecular weight $(\mathrm{MW})=2,000$; Sigma-Aldrich Co., $\mathrm{St}$ Louis, MO, USA), 3-(4,5-dimethylthiazolyl-2)-2,5-diphenyltetrazolium bromide (MTT; Nanjing Jiancheng Bioengineering Institute, Nanjing, China), dimethyl sulfoxide (DMSO;
Sigma Chemicals, Perth, Australia), and Dulbecco's Modified Eagle's Medium (DMEM; Thermo Fisher Scientific, Waltham, MA, USA) were used in this study. Other reagents were of analytical grade and were used without further purification.

\section{Synthesis of N-CDs}

The procedure for preparation of the N-CDs is illustrated in Figure 1. Briefly, $1.26 \mathrm{~g}$ of CA was dissolved in $10 \mathrm{~mL}$ of distilled water containing $40 \mathrm{mg}$ of PEI. The mixture was then transferred into a stainless steel autoclave with a Teflon liner of a 25-mL capacity (Tech Tai Beijing Technology Co, Ltd, Beijing, China) and heated at $100^{\circ} \mathrm{C}$ for $2 \mathrm{~h}$. After cooling to room temperature, the resulting light yellow solution was centrifuged at $15,000 \times g$ for $30 \mathrm{~min}$ to remove the large precipitate and agglomerated particles, followed by dialyzation against distilled water using a dialymksis bag (molecular weight cut-off $[\mathrm{MWCO}]=500$ ). The purified products were lyophilized and collected for further use.

\section{Characterization of N-CDs}

The morphology and size of the N-CDs were examined using transmission electron microscopy (TEM) with a JEM-2100F microscope (JEOL, Tokyo, Japan). The specimen for TEM was prepared by dropping an aqueous dispersion of N-CDs onto a copper grid-coated carbon film, which was subsequently dried under vacuum. Fourier transform infrared (FTIR) spectra were collected using the IR Prestige-21 spectrophotometer (Shimadzu, Kyoto, Japan). The X-ray photoelectron spectroscopy (XPS) spectra of the CDs were measured using an Axis Ultra Imaging Photoelectron Spectrometer (Kratos Analytical Ltd, Manchester, UK), using a monochromator of Al-K $\alpha$ as the source of excitation ( $h v$ $=1,486.7 \mathrm{eV}$ ), and the binding energy calibration was based on $\mathrm{C} 1 \mathrm{~s}$ at $284.8 \mathrm{eV}$. The X-ray diffraction (XRD) pattern was obtained using a Rigaku Ultima IV X-ray Diffractometer (Rigaku America, Woodlands, TX, USA), using $\mathrm{Cu} \cdot \mathrm{K} \alpha$

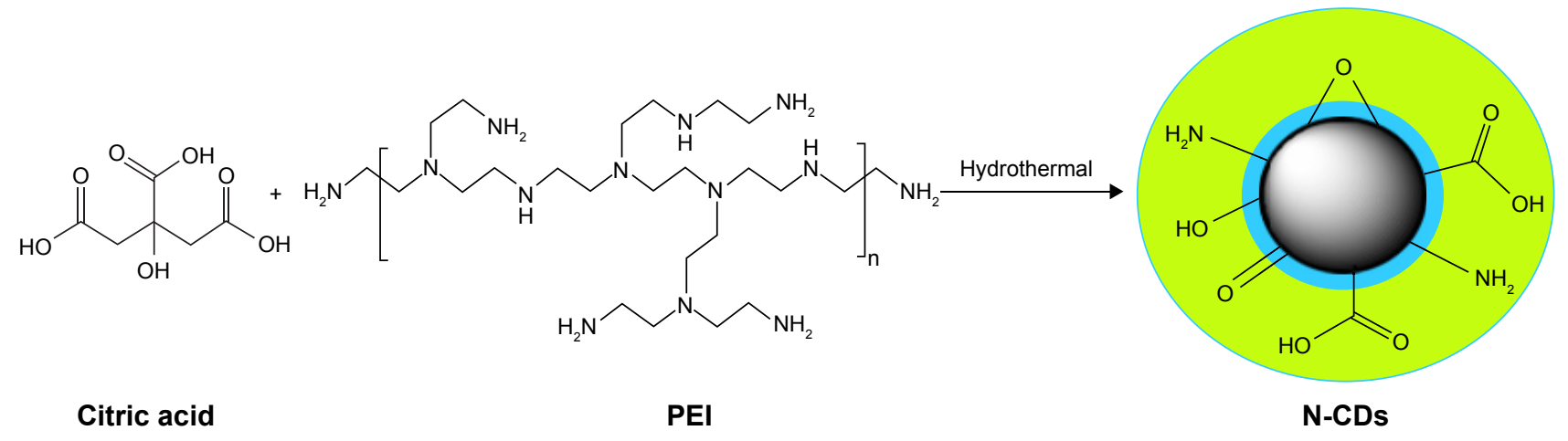

Figure I Schematic illustration of hydrothermal synthesis of N-CDs using citric acid and PEI.

Abbreviations: N-CDs, nitrogen-doped carbon dots; PEI, polyethylenimine. 
radiation $(\lambda=1.5405 \AA)$ at a voltage of $40 \mathrm{kV}$ and a current of $40 \mathrm{~mA}$ with $2 \theta$ scanning mode. The ultraviolet-visible (UV-Vis) absorption spectrum of the N-CDs was collected using a UV-2550 spectrophotometer (Shimadzu). The PL measurements were performed using an F-2500 spectrofluorophotometer (Hitachi Ltd., Tokyo, Japan) with a slit width of $2.5 \mathrm{~nm}$ for both excitation and emission.

\section{Measurement of QY}

QY $(\Phi)$ measurement was conducted according to a previously established procedure by comparing the integrated PL intensities. Quinine sulfate in $0.1 \mathrm{M} \mathrm{H}_{2} \mathrm{SO}_{4}(\mathrm{QY}=54 \%$ ) was chosen as a standard. ${ }^{19,20}$ The absorbance of the aqueous solutions of the N-CDs and quinine sulfate was kept $<0.10$ at $360 \mathrm{~nm}$. The QY was calculated using the following equation:

$$
\Phi_{\mathrm{X}}=\Phi_{\mathrm{ST}}\left(\frac{\operatorname{Grad}_{\mathrm{x}}}{\operatorname{Grad}_{\mathrm{sT}}}\right)\left(\frac{\eta_{\mathrm{X}}^{2}}{\eta_{\mathrm{ST}}^{2}}\right)
$$

where the subscripts ST and $\mathrm{X}$ denote the quinine standard and N-CDs, respectively; $\Phi$ is the QY, Grad is the gradient from the linear regression analysis; and $\eta$ is the refractive index of water (1.33).

\section{Cytotoxicity}

The cytotoxicity of the N-CDs was assessed using the MTT assay. 293T cells were seeded in a 96-well plate at a density of $2 \times 10^{4}$ cells/well and were incubated overnight at $37^{\circ} \mathrm{C}$ under $5 \% \mathrm{CO}_{2}$. Subsequently, the culture medium in each well was replaced with $100 \mu \mathrm{L}$ of fresh DMEM. Then, serial dilutions of N-CDs $(20 \mu \mathrm{L})$ were performed, resulting in a range of known concentrations in the treatment wells. After incubation for $24 \mathrm{~h}$, the medium containing the N-CDs was removed and replaced with $120 \mu \mathrm{L}$ of fresh medium containing $20 \mu \mathrm{L}$ of MTT, and the cells were incubated for another $4 \mathrm{~h}$. Finally, the entire medium was removed and $150 \mu \mathrm{L}$ of DMSO was added, followed by shaking for $15 \mathrm{~min}$. The absorbance of each well was measured at $490 \mathrm{~nm}$ using a Synergy HT Multi-Mode Microplate Reader (BioTek, Winooski, VT, USA) with pure DMSO as a blank. Non-treated cells (in DMEM) were used as a control, and the relative cell viability (mean \pm standard deviation [SD]) was expressed as

$$
\mathrm{Abs}_{\text {sample }} / \mathrm{Abs}_{\text {control }} \times 100 \% \text {. }
$$

\section{Confocal microscopy and imaging}

In total, $293 \mathrm{~T}$ cells were seeded in $35 \mathrm{~mm}$ cell culture plates at a density of $3 \times 10^{4}$ cells $/ \mathrm{mL}$ and were incubated at $37^{\circ} \mathrm{C}$ under $5 \% \mathrm{CO}_{2}$ for $24 \mathrm{~h}$. Then, $20 \mu \mathrm{L}$ of N-CDs $(1.5 \mathrm{mg} / \mathrm{mL})$ was added to each well. The real-time live-cell images were captured using Leica confocal laser scanning fluorescence microscopy (velocity 6-cell analysis system; PerkinElmer Inc., Waltham, MA, USA) for $12 \mathrm{~h}$ with a time interval of $30 \mathrm{~min}$. The fluorescence intensity in the real-time images of the 293T cells incubated with the N-CDs was analyzed using the ImageJ image processing software (Bethesda, MD, USA).

\section{Establishment of an in vitro BBB model}

Primary rat microvascular endothelial cells and astrocytes were isolated, and an in vitro biomimetic BBB model was established as described in previous studies. ${ }^{16,21,22}$ Briefly, Millipore-R transwells (EMD Millipore, Billerica, MA, USA) were coated with $100 \mu \mathrm{L}$ of poly-L-lysine for $3 \mathrm{~h}$, washed twice with phosphate-buffered saline (PBS), and dried before use in a 24-well plate. The backsides of the transwells were inverted and seeded with $100 \mu \mathrm{L}$ of astrocytes at a density of $8 \times 10^{5}$ cells $/ \mathrm{mL}$ and incubated for $1.5 \mathrm{~h}$ at $37^{\circ} \mathrm{C}$ under $5 \% \mathrm{CO}_{2}$, after which the astrocytes had successfully attached to the transwell surface. Then, the transwell was reinverted, and $600 \mu \mathrm{L}$ of DMEM medium containing fetal cattle serum was added to the wells and cultured for 3 days. On the fourth day, $300 \mu \mathrm{L}$ of ECM medium containing fetal cattle serum was added to the transwells, and then rat brain microvascular endothelial cells (RBMEC) at a density of $1.5 \times 10^{6}$ cells $/ \mathrm{mL}(100 \mu \mathrm{L})$ were seeded. The transendothelial electrical resistance (TEER) of the RBMEC monolayers in the presence of astrocytes was measured $\sim 10$ days later using a Millicell-ERS (EMD Millipore). The experiments were performed when the TEER reached $200 \Omega \cdot \mathrm{cm}^{2}$.

\section{Assessment of the BBB-penetration ability of the $\mathrm{N}-\mathrm{CD}$ s}

The N-CDs at different concentrations $(0.25$ and $0.5 \mathrm{mg} / \mathrm{mL})$ in PBS were investigated using the in vitro BBB model $(n=3)$. After predetermined incubation intervals, the fluid in the lower chamber containing the N-CDs, which had penetrated the in vitro biomimetic BBB, was withdrawn and transferred to a 96-well plate for measuring the fluorescent signal at 360-nm excitation and 452-nm emission wavelengths (Gemini EM Fluorescence Microplate Reader; Molecular Devices, Sunnyvale, CA, USA). The lower chamber of the transwell was then refilled with fresh PBS.

\section{Statistical analysis}

Statistical analysis was performed using the paired Student's $t$-test and one-way analysis of variance. $P$-values $<0.05$ were considered to indicate significant differences between the groups. The values were expressed as mean $\pm \mathrm{SD}$. 


\section{Results and discussion}

\section{Characterization}

In this study, the N-CDs were synthesized using a one-pot hydrothermal treatment with CA in the presence of PEI. Although a detailed mechanism remains unclear, it was proposed that first the reactions of CA and PEI form a large network due to the existence of multiple functional groups at both the precursor molecules and then the polymerized network carbonizes at the elevated temperature and results in the formation of the N-CDs. Meanwhile, PEI as the surface passivated agent was covalently attached to the N-CDs by the amide linkages (-NHCO-), derived from the thermal dehydration of ammonium carboxylate moieties between the $-\mathrm{COOH}$ groups of $\mathrm{N}-\mathrm{CD}$ s and the $-\mathrm{NH}_{2}$ groups of PEI. The simultaneously progressive carbonization and functionalization of PEI lead to the formation of a hydrophilic photoluminescent N-CD.

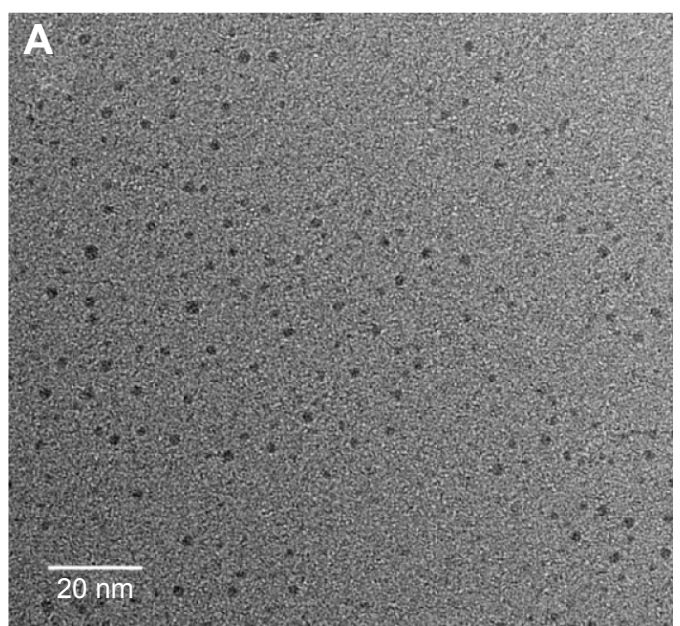

C

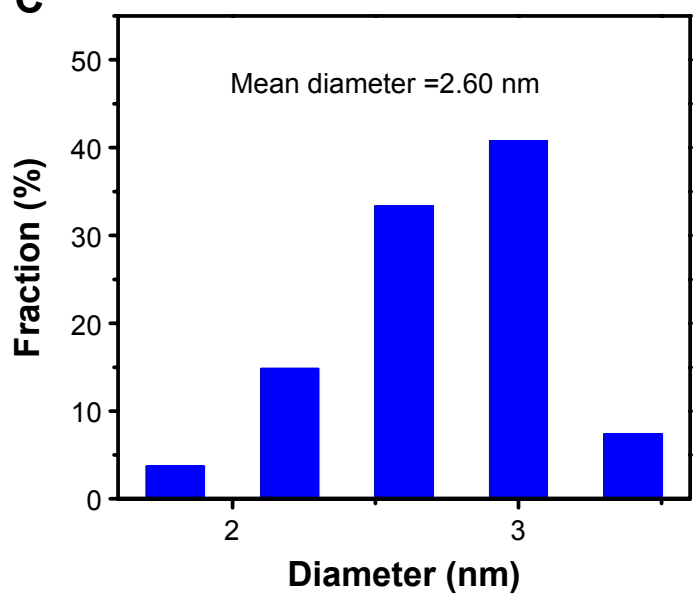

The size and morphology of the hydrothermally synthesized N-CDs were examined using TEM. The TEM images (Figure 2A and B) reveal that the N-CDs are monodispersed and form semispherical shape with an approximate diameter of $2.6 \mathrm{~nm}$, which is obtained via averaging the diameters of $50 \mathrm{~N}-\mathrm{CDs}$ (Figure 2C). The results indicated that there is good coincidence between the average hydrodynamic diameter $(\sim 2.99 \mathrm{~nm})$ and the dynamic light scattering analysis (Figure 2D). Moreover, it was observed from high-resolution transmission electron microscopy (HRTEM) images of single N-CD particles that the in-plane lattice spacing of the N-CDs is $\sim 0.22 \mathrm{~nm}$ (inset, Figure 2B). Additionally, the XRD pattern of the as-prepared N-CDs (Figure 3A) shows a broad diffraction peak centered at $2 \theta=20^{\circ}$, which is attributed to the turbostratic carbon phase.

Next, the surface functional groups and chemical composition of the N-CDs were identified using FTIR (Figure 3B).

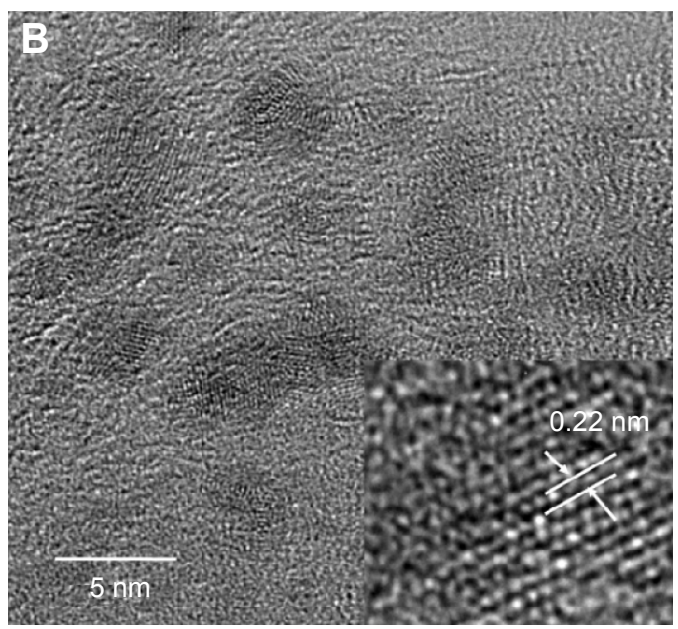

D

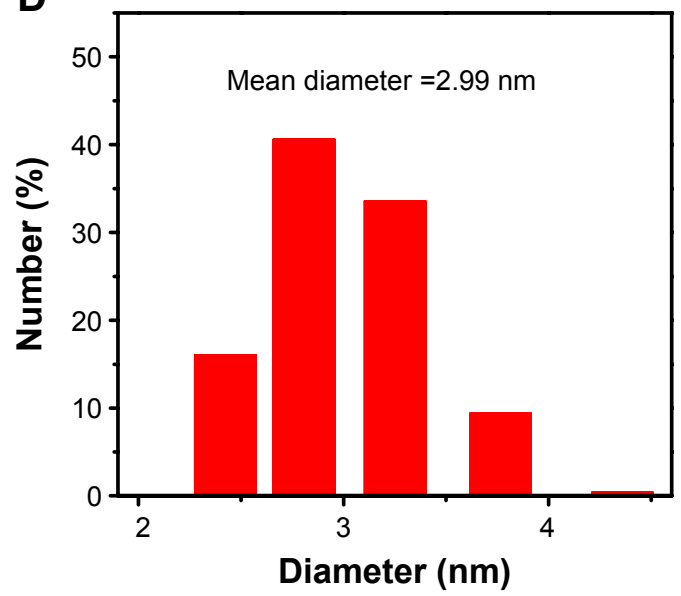

Figure 2 The image and size distribution of $\mathrm{N}-\mathrm{CDs}$.

Notes: The TEM image (A) and size distribution (C) of N-CDs. An HRTEM image of a single N-CD particle. The inset shows one in-plane lattice spacing of the N-CDs, as indicated by arrows (B). The size distribution of $\mathrm{N}-\mathrm{CD}$ s measured by DLS analysis (D).

Abbreviations: DLS, dynamic light scattering; HRTEM, high-resolution transmission electron microscopy; N-CDs, nitrogen-doped carbon dots; TEM, transmission electron microscopy. 

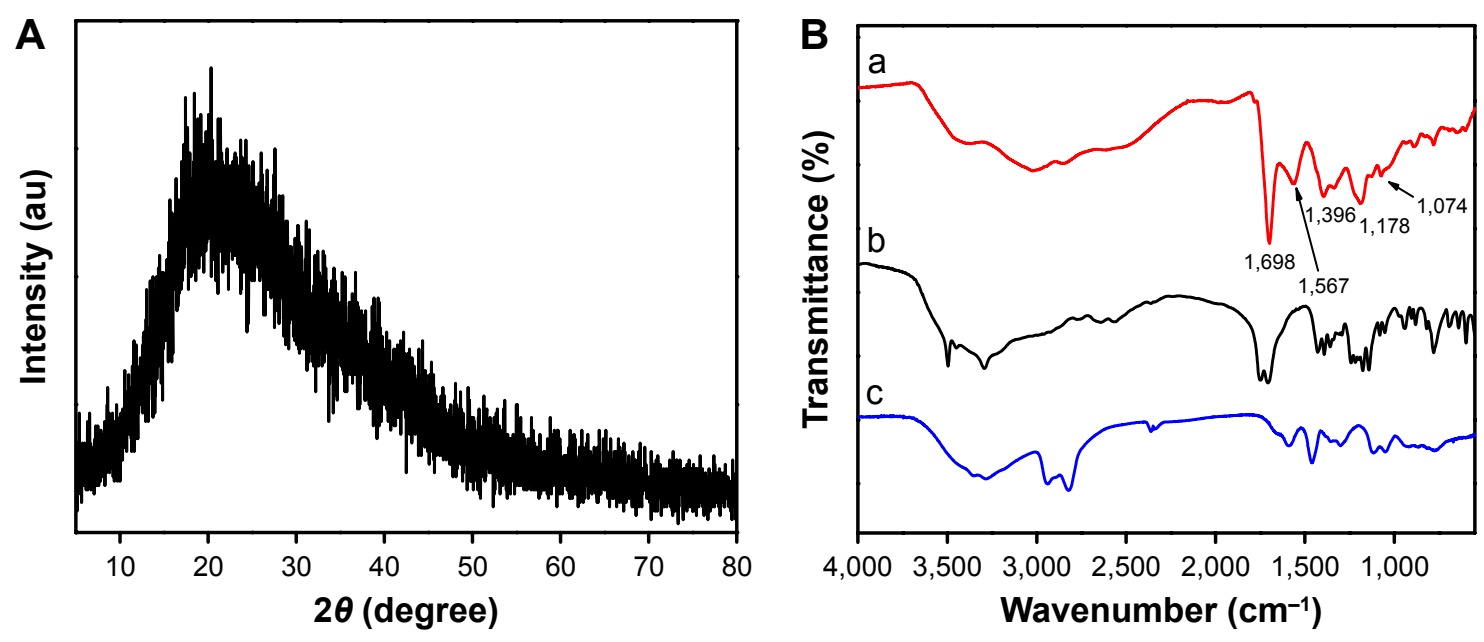

Figure 3 The XRD pattern and FTIR spectra of N-CDs.

Notes: (A) XRD pattern of N-CDs. (B) FTIR spectra of N-CDs (a), CA (b), and PEI (c).

Abbreviations: au, arbitrary units; CA, citric acid; FTIR, Fourier transform infrared; N-CDs, nitrogen-doped carbon dots; PEI, polyethylenimine; XRD, X-ray diffraction.

The FTIR spectra of CA and PEI are provided for comparison. The FTIR spectra of the N-CDs are obviously different from those of the PEI and CA, suggesting that the N-CDs are successfully formed. Specifically, the bands at 1,396 and $1,074 \mathrm{~cm}^{-1}$ are attributed to the stretching and bending vibrations of $\mathrm{N}-\mathrm{H}$. A sharp band at $1,698 \mathrm{~cm}^{-1}$ is attributed to $\mathrm{C}=\mathrm{O}$ stretching. In addition, a band at $1,187 \mathrm{~cm}^{-1}$ is apparent, which is usually found in oxidized carbons and has been assigned to $\mathrm{C}-\mathrm{O}$ stretching. The band at $1,380 \mathrm{~cm}^{-1}$ reveals the presence of $\mathrm{CH}_{2}$ in the $\mathrm{N}-\mathrm{CDs}$. Meanwhile, the carbogenic core of the N-CDs results in an infrared (IR) band at $1,567 \mathrm{~cm}^{-1}$, which is attributed to $\mathrm{C}=\mathrm{C}$ stretching.

The surface functional groups of the N-CDs were further investigated using XPS. The XPS survey spectrum (Figure 4A) shows characteristic peaks corresponding to C1s (284.89 eV), O1s (531.84 eV), and N1s (401.32 eV), confirming that the $\mathrm{N}-\mathrm{CD}$ are mainly composed of $\mathrm{C}, \mathrm{O}$, and $\mathrm{N}$ elements. The high-resolution O1s XPS spectrum (Figure 4B) is dominated by one peak attributed to $\mathrm{C}-\mathrm{O}$. The high-resolution N1s XPS spectrum (Figure 4C) exhibits two peaks located at 399.29 and $401.32 \mathrm{eV}$, which can be attributed to $\mathrm{C}=\mathrm{C}-\mathrm{N}$ and $\mathrm{O}=\mathrm{C}-\mathrm{N}$, respectively. The $\mathrm{C} 1 \mathrm{~s}$ high-resolution XPS spectrum (Figure 4D) shows three peaks assigned to the carbon atoms in $\mathrm{C}=\mathrm{C}(284.80 \mathrm{eV})$, $\mathrm{C}=\mathrm{O}(286.12 \mathrm{eV})$, and $\mathrm{COOH}(288.42 \mathrm{eV})$.

\section{PL properties}

The optical properties of the N-CDs were investigated using UV-Vis absorption and PL spectra. The UV-Vis absorption spectrum of the as-prepared N-CDs demonstrates peaks at 360 and $235 \mathrm{~nm}$ (Figure 5A). Specifically, the peak at $235 \mathrm{~nm}$ is ascribed to the $\pi-\pi^{*}$ transition of aromatic domains in the carbonized core of the N-CDs, while the peak at $360 \mathrm{~nm}$ is attributed to the $n-\pi *$ transition of surface functional groups, such as $\mathrm{C}=\mathrm{O}$. The PEI-passivated N-CDs could be easily dispersed in water, forming a transparent yellow dispersion. Under UV irradiation, the N-CD aqueous dispersion emitted a blue luminescence (inset, Figure 5A). Accordingly, the PL emission spectrum showed a peak at $\sim 452 \mathrm{~nm}$ under excitation at $360 \mathrm{~nm}$ (Figure $5 \mathrm{~A}$ ). The QY of the N-CDs was as high as $51 \%$ (Figure S1). Moreover, these N-CDs demonstrated good photostability under continuous excitation at $360 \mathrm{~nm}$ (Figure S2).

A detailed study of this behavior was implemented by varying the excitation wavelength from 300 to $420 \mathrm{~nm}$, in $20 \mathrm{~nm}$ increments. It was determined that the emission of the N-CDs is excitation independent, that is, the position of the emission peak is consistent (up to $460 \mathrm{~nm}$ ). However, the intensity of the photoluminescence decreased with an increase in the excitation wavelength. The observed excitation-independent emission thereby indicates a relatively uniform emission at the N-CD surface (Figure 5B).

\section{Cytotoxicity}

Aside from a high QY and photostability, another prerequisite for N-CDs in bio-applications is low cytotoxicity. To assess their cytotoxicity, the $293 \mathrm{~T}$ cells were exposed to the N-CDs at a series of concentrations for $24 \mathrm{~h}$ and cell viability was examined using the MTT assay. The viability of $293 \mathrm{~T}$ cells exposed to the N-CDs was still $>80 \%$ compared with the control cells, even at a concentration as high as $5 \mathrm{mg} / \mathrm{mL}$ (Figure 6), indicating that the $\mathrm{N}-\mathrm{CD}$ possess a low cytotoxicity. 
A

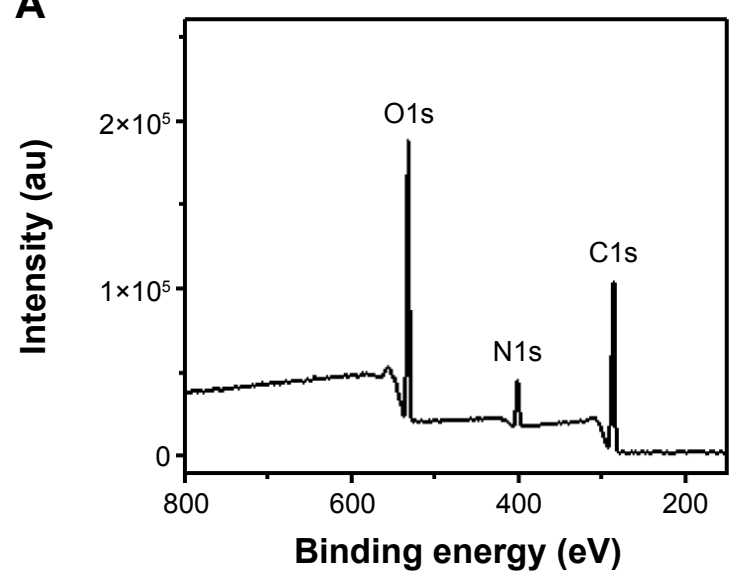

C

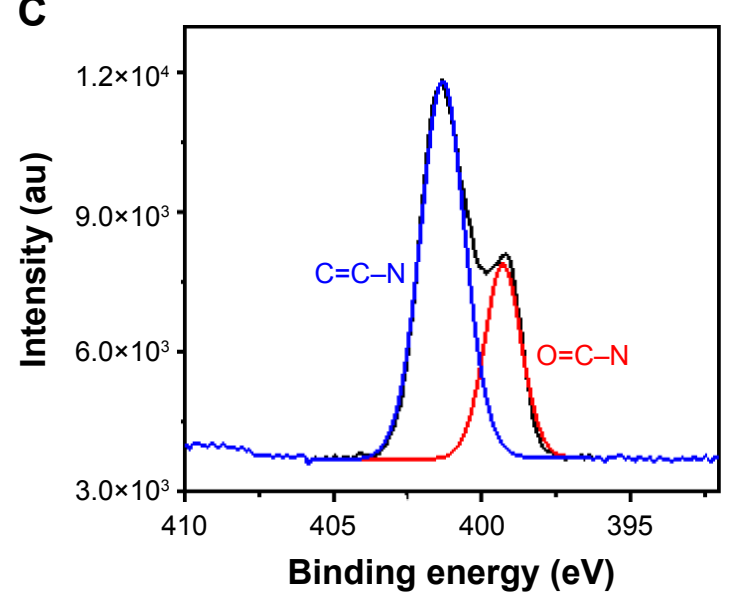

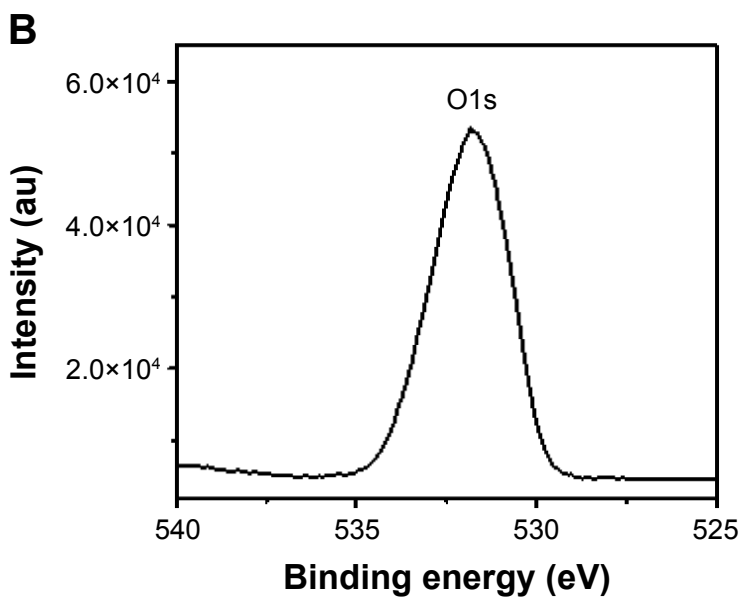

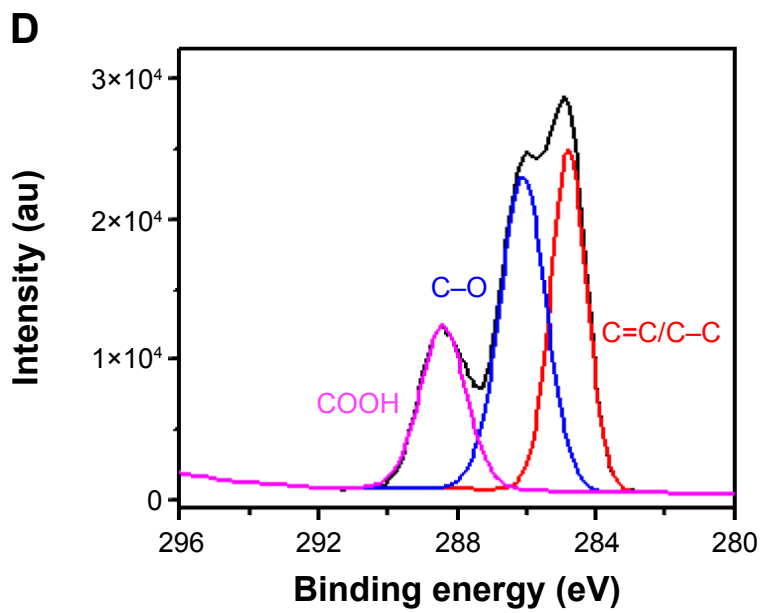

Figure 4 Compositions of N-CDs.

Notes: (A) XPS survey spectra, (B) XPS OIs spectra, (C) XPS NIs spectra, and (D) XPS CIs spectra of N-CDs.

Abbreviations: au, arbitrary units; N-CDs, nitrogen-doped carbon dots; XPS, X-ray photoelectron spectroscopy.

A

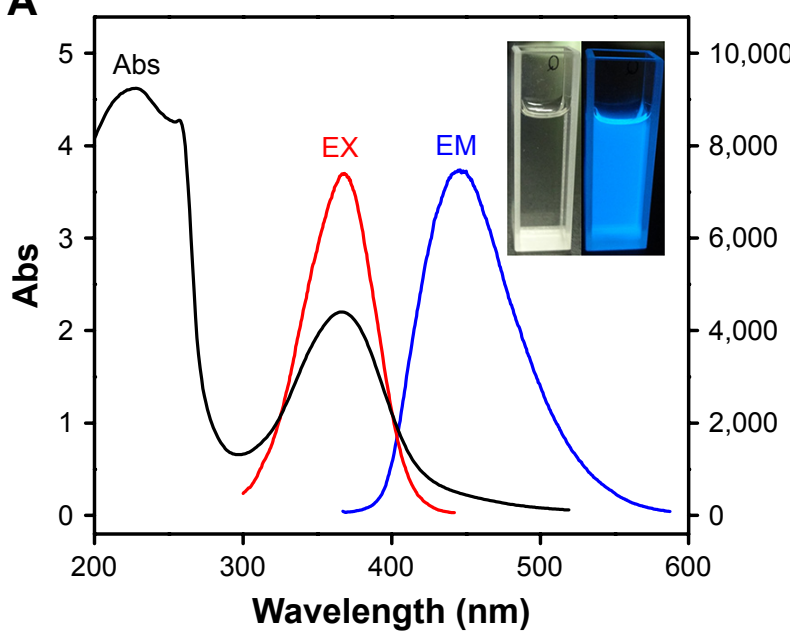

B

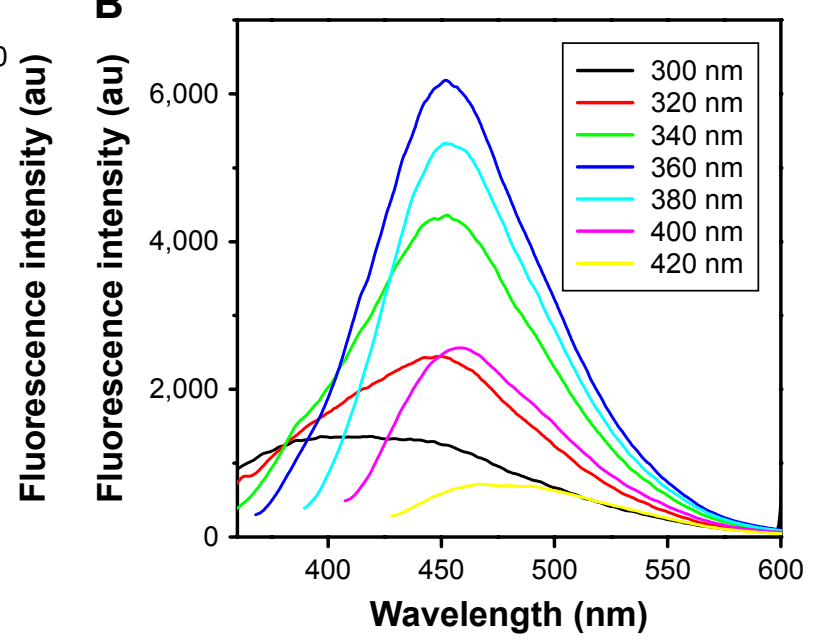

Figure 5 The optical properties of N-CDs.

Notes: (A) UV-Vis absorption, PL excitation $\left(\lambda_{\text {ex }}=360 \mathrm{~nm}\right)$, and PL emission $\left(\lambda_{\text {em }}=452 \mathrm{~nm}\right)$ spectra of $N-C D$ s. The inset shows the photos of $N-C D$ dispersion under daylight (left) and $365 \mathrm{~nm}$ UV light (right). (B) PL emission spectra of N-CDs at different excitation wavelengths.

Abbreviations: Abs, absorbance; au, arbitrary units; EM, emission wavelength; EX, excitation wavelength; N-CDs, nitrogen-doped carbon dots; PL, photoluminescence; UV-Vis, ultraviolet-visible. 


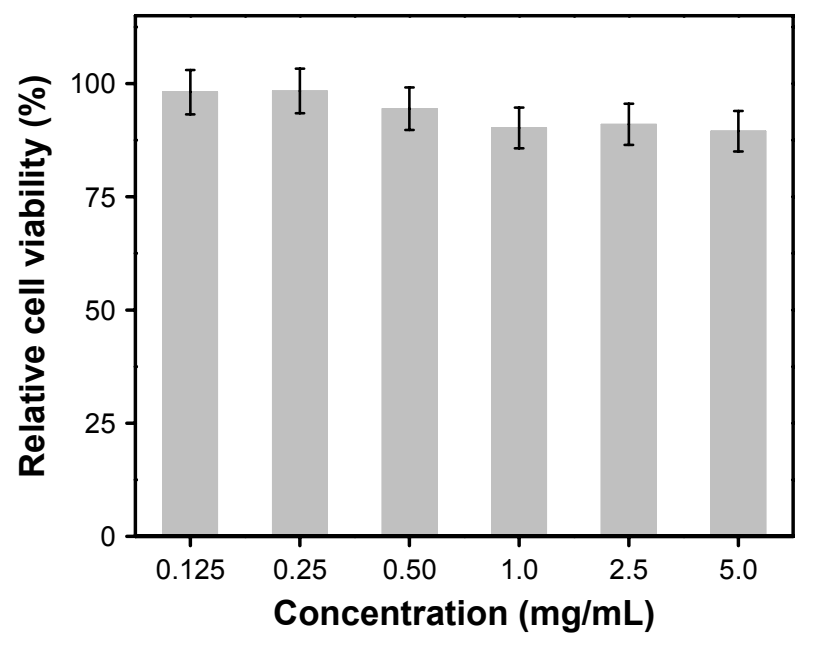

Figure 6 Viability of 293T cells via incubation with N-CDs at different concentrations for $24 \mathrm{~h}$ (mean $\pm S D, \mathrm{n}=3$ ).

Abbreviations: $\mathrm{N}-\mathrm{CD}$, nitrogen-doped carbon dots; SD, standard deviation.

\section{In vitro real-time live-cell imaging}

In light of their high QY, excellent photostability, and low cytotoxicity, the N-CDs are thereby applicable as optical nanoprobes for in vitro real-time live-cell imaging. Timelapse laser scanning confocal images were acquired every $30 \mathrm{~min}$ for $12 \mathrm{~h}$ and then combined to form a cell video
(Video S1). The representative time-lapse images collected at $0,1,2,4,8$, and $12 \mathrm{~h}$ are shown in Figures 7 and S3. It was showed that, with an increase in incubation time, the N-CDs yielded increasingly stronger blue fluorescence signals, suggesting that a gradually underwent uptake existed via the cells. These signals could persist up to $12 \mathrm{~h}$, indicating that these N-CDs, with a high QY and photostability, are promising optical nanoprobes for real-time cell imaging and tracking. The increase in fluorescence intensity against the control cells was also confirmed by confocal microscopy images (Figure S4).

Furthermore, it was observed that after $4 \mathrm{~h}$ of incubation, the N-CDs were primarily located around the nuclear region. However, after $8 \mathrm{~h}$ of incubation, a number of the N-CDs entered the nucleus, suggesting that they could traverse the nuclear membrane. In addition, no notable change in the morphology of the cells was observed during the entire period of imaging, further confirming the low cytotoxicity of the N-CDs.

\section{BBB-penetration ability of the N-CDs}

The BBB is the most important barrier system in the central nervous system. ${ }^{23}$ This barrier has a high transendothelial
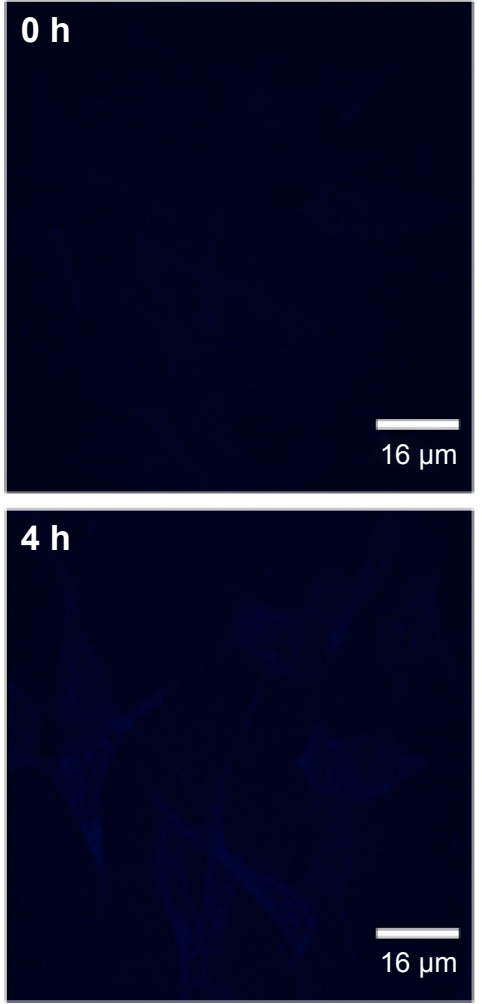

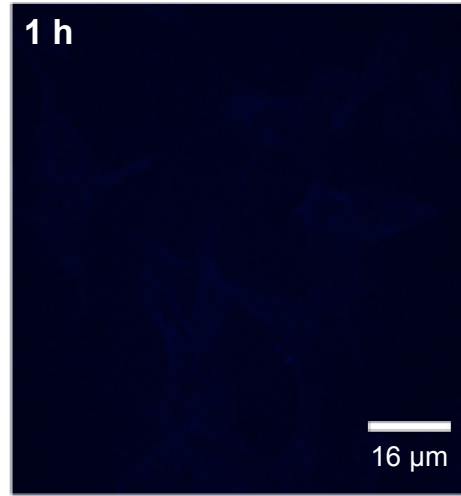

\section{$8 \mathrm{~h}$}

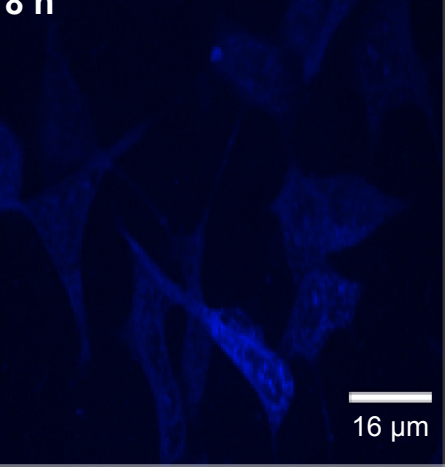

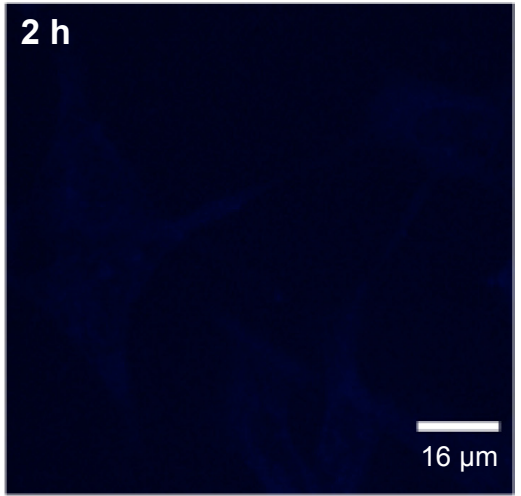

$12 \mathrm{~h}$

Figure 7 In vitro real-time (0-12 h) imaging of live 293T cells using the N-CDs. Abbreviation: N-CDs, nitrogen-doped carbon dots. 


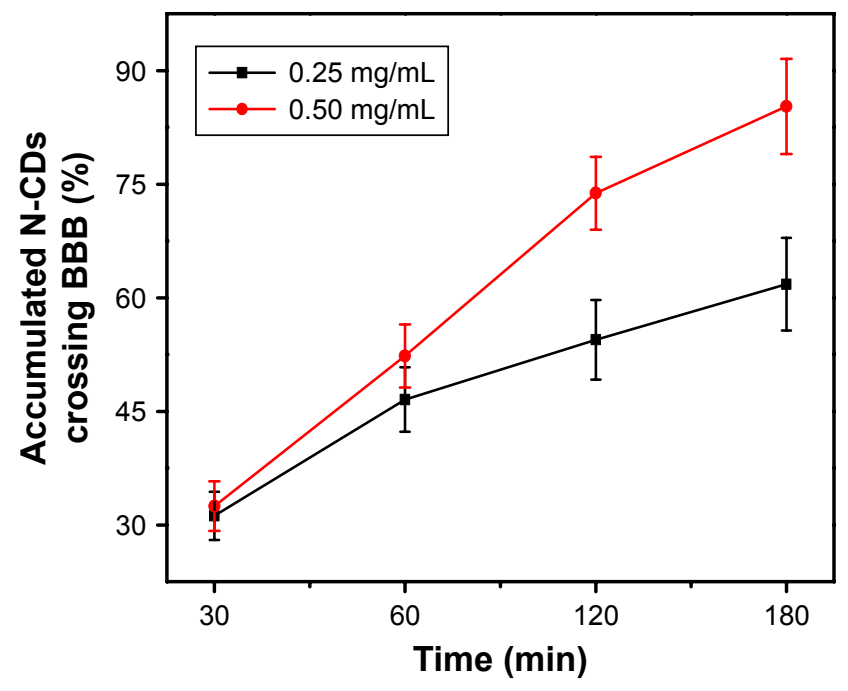

Figure 8 Accumulated percentages of $\mathrm{N}$-CDs that crossed the BBB.

Note: All data are expressed as mean $\pm S D(n=3)$.

Abbreviations: $\mathrm{BBB}$, blood-brain barrier; $\mathrm{N}-\mathrm{CDs}$, nitrogen-doped carbon dots; $\mathrm{SD}$, standard deviation.

resistance that prohibits the diffusion and transport of some therapeutic agents from the blood to the brain. However, the tight junctions in the BBB have gaps of within 4-6 nm. Thus, it is possible that nanoparticles with a size $<4 \mathrm{~nm}$ could pass through the BBB via such gaps. Given that the size of the N-CDs is $\sim 2.6 \mathrm{~nm}$, their ability to cross the BBB was investigated using a biomimetic BBB model, which was constructed using RBMECs and astrocytes, corresponding to the anatomical situation in brain capillaries. The high TEER value obtained demonstrates that the co-culture BBB model can be successfully used to mimic the in vivo BBB environment (Figure S5). The BBB-penetration ability of the $\mathrm{N}-\mathrm{CDs}$ was evaluated via monitoring the fluorescence intensity of BBB-penetrating N-CDs, which accumulated in the lower chamber of a BBB-biomimetic transwell. As shown in Figure 8, the N-CDs are able to cross the BBB in a concentration- and time-dependent manner, which is attributed to their small size. Moreover, the cationic PEI on the surface of the N-CDs could facilitate BBB penetration as well.

\section{Conclusion}

The N-CDs were fabricated using a one-pot hydrothermal method using CA as the carbon source and PEI as a simultaneous nitrogen dopant and surface passivation agent. The PEI-passivated N-CDs emit a bright blue PL under UV excitation with a high QY of 51\%. This taken together with their excellent photostability and low cytotoxicity makes the $\mathrm{N}-\mathrm{CDs}$ promising optical nanoprobes for real-time live-cell imaging. Moreover, their small size and the surface positive charge rendered by the PEI endow them with the ability to cross the BBB, making them potentially useful in traceable drug delivery in brain disorders.

\section{Acknowledgments}

The authors gratefully acknowledge financial support from the National Foundation of Natural Sciences of China (81173121 and 81573683), Beijing Natural Science Foundation (7162023), and Beijing Laboratory for Biomedical Detection Technology and Instrument (PXM2014-014226-000021) under the jurisdiction of the Beijing Municipality of China, Central Public-interest Scientific Institution Basal Research Fund (2015 CZ-29).

\section{Disclosure}

The authors report no conflicts of interest in this work.

\section{References}

1. Zhu SJ, Meng QN, Wang L, et al. Highly photoluminescent carbon dots for multicolor patterning, sensors, and bioimaging. Angew Chem Int Ed Engl. 2013;52(14):3953-3957.

2. Li J, He Z, Guo CR, Wang LP, Xu SK. Synthesis of carbon nanohorns/ chitosan/quantum dots nanocomposite and its applications in cells labeling and in vivo imaging. J Lumin. 2014;145:74-80.

3. Kong B, Zhu AW, Ding CQ, Zhao XM, Li B, Tian Y. Carbon dot-based inorganic-organic nanosystem for two-photon imaging and biosensing of $\mathrm{pH}$ variation in living cells and tissues. Adv Mater. 2012;24(43): 5844-5848.

4. Tan H, Liu WX, Gong B, et al. Unique dual functions for carbon dots in emulsion preparations: costabilization and fluorescence probing. Langmuir. 2015;31(35):9537-9545.

5. Wang L, Li BQ, Xu F, et al. High-yield synthesis of strong photoluminescent $\mathrm{N}$-doped carbon nanodots derived from hydrosoluble chitosan for mercury ion sensing via smartphone APP. Biosens Bioelectron. 2016; $79: 1-8$.

6. Guo SS, Yang M, Chen M, et al. Bioinspired synthesis of fluorescent calcium carbonate/carbon dot hybrid composites. Dalton Trans. 2015; 44(17):8232-8237.

7. Tan MQ, Li XT, Wu H, Wang BB, Wu J. N-doped carbon dots derived from bovine serum albumin and formic acid with one- and two-photon fluorescence for live cell nuclear imaging. Colloids SurfB Biointerfaces. 2015;136:141-149.

8. Konwar A, Gogoi N, Majumdar G, Chowdhury D. Green chitosancarbon dots nanocomposite hydrogel film with superior properties. Carbohydr Polym. 2015;115:238-245.

9. Wang N, Wang Y, Guo T, Yang T, Chen M, Wang J. Green preparation of carbon dots with papaya as carbon source for effective fluorescent sensing of Iron (III) and Escherichia coli. Biosens Bioelectron. 2016; 85:68-75.

10. Ruan SB, Zhu BY, Zhang HJ, et al. A simple one-step method for preparation of fluorescent carbon nanospheres and the potential application in cell organelles imaging. J Colloid Interface Sci. 2014;422:25-29.

11. Han BF, Wang WX, Wu HY, et al. Polyethyleneimine modified fluorescent carbon dots and their application in cell labeling. Colloids Surf B Biointerfaces. 2012;100:209-214.

12. Barati A, Shamsipur M, Arkan E, Hosseinzadeh L, Abdollahi H. Synthesis of biocompatible and highly photoluminescent nitrogen doped carbon dots from lime: analytical applications and optimization using response surface methodology. Mater Sci Eng C Mater Biol Appl. 2015;47:325-332. 
13. Yusof SR, Avdeef A, Abbott NJ. In vitro porcine blood-brain barrier model for permeability studies: pCEL-X software pK(a)(FLUX) method for aqueous boundary layer correction and detailed data analysis. Eur J Pharm Sci. 2014;65:98-111.

14. Wang QL, Huang XX, Long YJ, et al. Hollow luminescent carbon dots for drug delivery. Carbon. 2013;59:192-199.

15. Li SH, Leblanc RM. Aggregation of Insulin at the Interface. J Phys Chem B. 2014;118(5):1181-1188.

16. Cai Q, Wang L, Deng G, Liu JH, Chen QX, Chen ZB. Systemic delivery to central nervous system by engineered PLGA nanoparticles. Am J Transl Res. 2016;8(2):749-764.

17. Bilensoy E. Cationic nanoparticles for cancer therapy. Expert Opin Drug Deliv. 2010;7(7):795-809.

18. Xie J, Lee S, Chen XY. Nanoparticle-based theranostic agents. $A d v$ Drug Deliv Rev. 2010;62(11):1064-1079.
19. Guan WW, Gu W, Ye L, et al. Microwave-assisted polyol synthesis of carbon nitride dots from folic acid for cell imaging. Int J Nanomedicine. 2014;9:5071-5078.

20. Guo SS, Lu SS, Xu PX, et al. Biomimetic synthesis of needle-like fluorescent calcium phosphate/carbon dot hybrid composites for cell labeling and copper ion detection. Dalton Trans. 2016;45(18):7665-7671.

21. Garcia-Garcia E, Gil S, Andrieux K, et al. A relevant in vitro rat model for the evaluation of blood-brain barrier translocation of nanoparticles. Cell Mol Life Sci. 2005;62(12):1400-1408.

22. Xu LM, Dan M, Shao AL, et al. Silver nanoparticles induce tight junction disruption and astrocyte neurotoxicity in a rat blood-brain barrier primary triple coculture model. Int J Nanomedicine. 2015;10:6105-6119.

23. Yang YY, Bai L, Li XR, et al. Transport of active flavonoids, based on cytotoxicity and lipophilicity: an evaluation using the blood-brain barrier cell and Caco-2 cell models. Toxicol In Vitro. 2014;28(3):388-396. 


\section{Supplementary materials}

Video SI N-CDs as optical nanoprobes for in vitro real-time live-cell imaging.

Abbreviation: N-CDs, nitrogen-doped carbon dots.

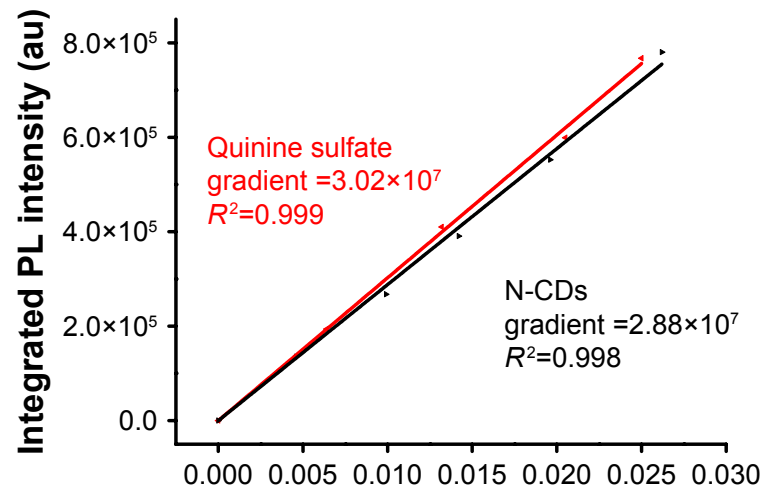

Abs

Figure SI QY measurement of N-CDs using quinine sulfate as the reference.

Abbreviations: Abs, absorbance; au, arbitrary units; N-CDs, nitrogen-doped carbon dots; PL, photoluminescence; QY, quantum yield.

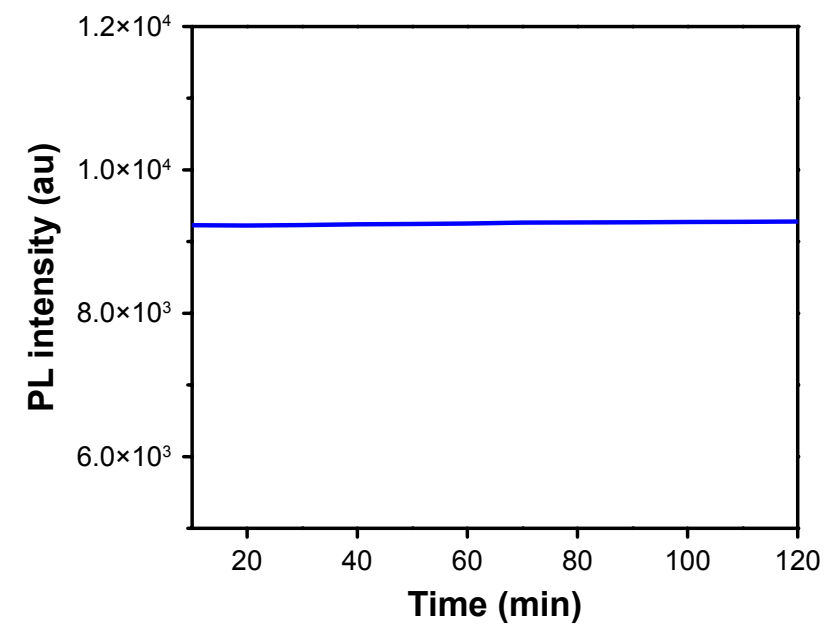

Figure S2 PL intensity of N-CDs against excitation time $\left(\lambda_{\text {ex }}=360 \mathrm{~nm}\right)$.

Abbreviations: au, arbitrary units; N-CDs, nitrogen-doped carbon dots; PL, photoluminescence.

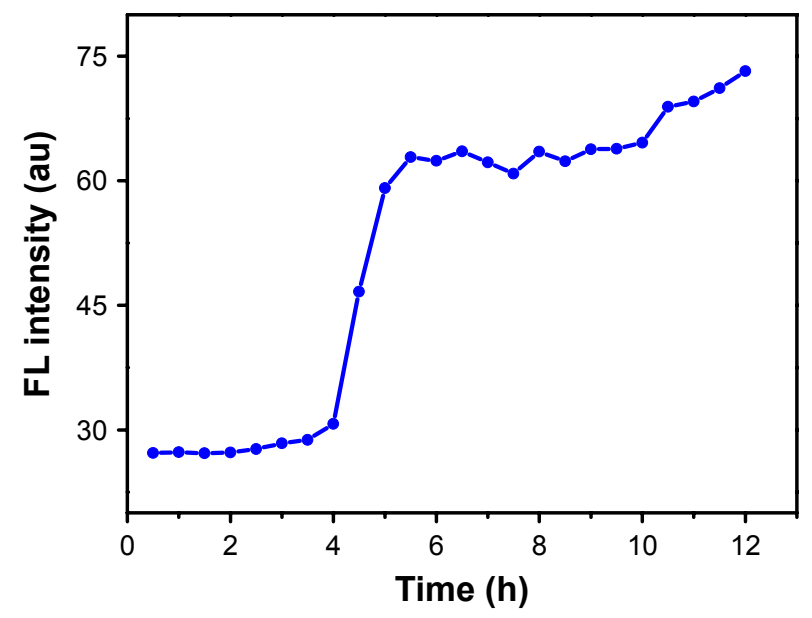

Figure S3 FL intensity forms real-time cell imaging of $\mathrm{N}-\mathrm{CDs}$ for $12 \mathrm{~h}$ with a 30 -min interval. Abbreviations: au, arbitrary units; FL, fluorescence; N-CDs, nitrogen-doped carbon dots. 

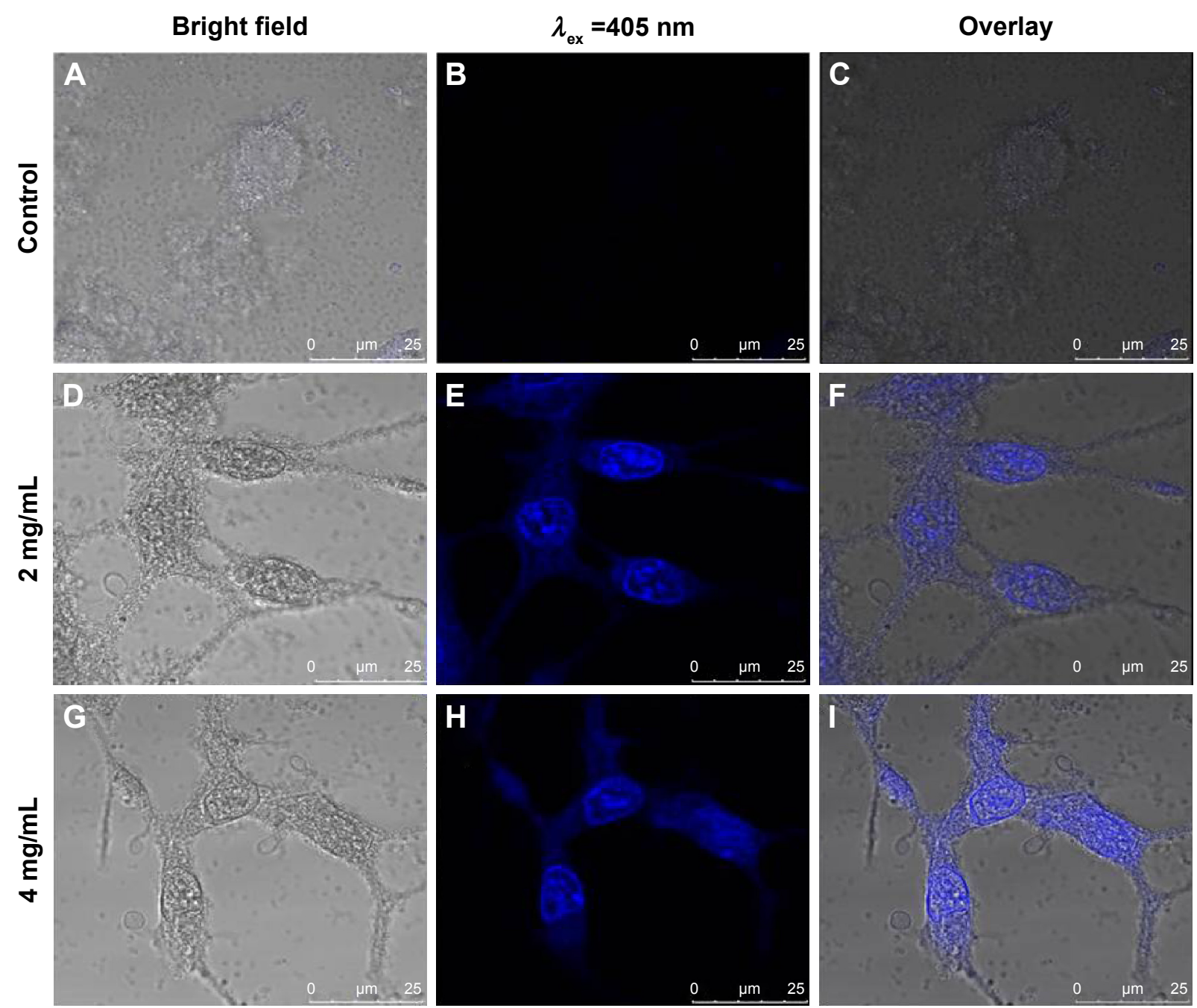

Figure S4 Confocal laser scanning microscopy images of $293 \mathrm{~T}$ cells incubated with $\mathrm{N}-\mathrm{CDs}$ at different dosages $(0,2$, and $4 \mathrm{mg} / \mathrm{mL})$.

Abbreviation: $\mathrm{N}-\mathrm{CD}$, nitrogen-doped carbon dots.

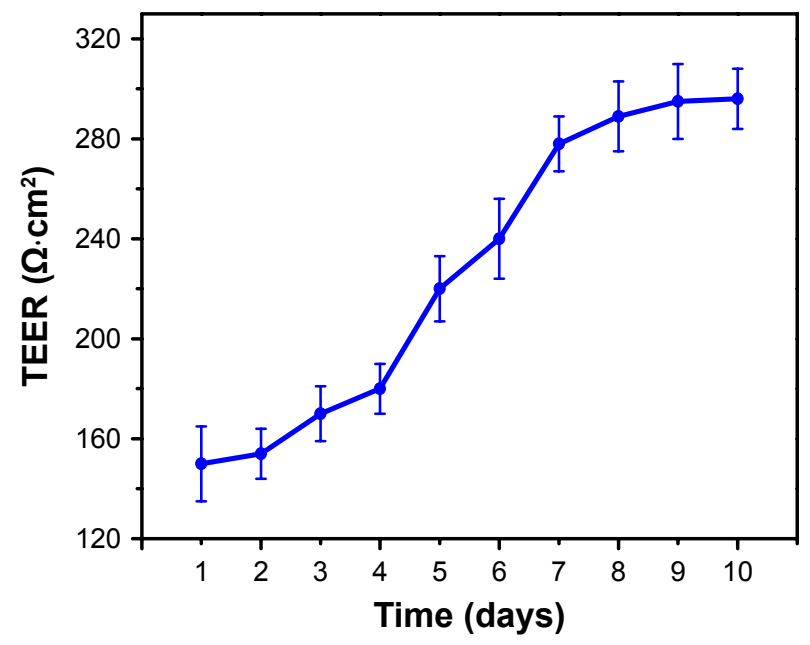

Figure S5 TEER values of rat brain endothelial cell monolayers in monoculture and coculture with astrocytes for 10 days in transwells after coated with poly-L-lysine. Note: All data are expressed as mean $\pm S D(n=3-4)$.

Abbreviations: SD, standard deviation; TEER, transendothelial electrical resistance. 


\section{Publish your work in this journal}

The International Journal of Nanomedicine is an international, peerreviewed journal focusing on the application of nanotechnology in diagnostics, therapeutics, and drug delivery systems throughout the biomedical field. This journal is indexed on PubMed Central, MedLine, CAS, SciSearch $\AA$, Current Contents $\AA /$ Clinical Medicine,

Journal Citation Reports/Science Edition, EMBase, Scopus and the Elsevier Bibliographic databases. The manuscript management system is completely online and includes a very quick and fair peer-review system, which is all easy to use. Visit http://www.dovepress.com/ testimonials.php to read real quotes from published authors.

Submit your manuscript here: http://www.dovepress.com/international-journal-of-nanomedicine-journal 